\title{
General Psychiatry Amplitude of low-frequency fluctuations on Alzheimer's disease with depression: evidence from resting-state fMRI
}

To cite: Mu Y, Li Y, Zhang Q, et al. Amplitude of low-frequency fluctuations on Alzheimer's disease with depression: evidence from resting-state fMRI. General Psychiatry 2020;33:e100147. doi:10.1136/ gpsych-2019-100147

- Additional material is published online only. To view please visit the journal online (http://dx.doi.org/10.1136/ gpsych-2019-100147).

Received 26 September 2019 Revised 20 May 2020 Accepted 03 June 2020

Check for updates

(C) Author(s) (or their employer(s)) 2020. Re-use permitted under CC BY-NC. No commercial re-use. See rights and permissions. Published by BMJ.

For numbered affiliations see end of article.

Correspondence to Dr Xiaoyun Guo; xiaoyunguo@163.com

Dr Maosheng Xu; xums166@zcmu.edu.cn

\author{
Yuzhu Mu, ${ }^{1}$ Yumei Li, ${ }^{2}$ Qi Zhang, ${ }^{3}$ Zhongxiang Ding, ${ }^{4}$ Mei Wang, ${ }^{4}$ Xingguang Luo, ${ }^{5}$ \\ Xiaoyun Guo, ${ }^{6}$ Maosheng $\mathrm{Xu}^{3}$
}

\section{ABSTRACT}

Background The prevalence of Alzheimer's disease (AD) comorbid with depression is common. However, the mechanisms of $A D$ with depression remain unclear. Aims To investigate the regional alterations of brain activity of $A D$ with depression in resting-state functional magnetic resonance imaging (rs-fMRI).

Methods 154 patients with $A D$ who met the inclusion criteria were recruited from the Zhejiang Provincial People's Hospital from October 2014 to October 2016. According to whether the core symptoms of depression were present, patients were divided into two groups, 22 patients with $A D$ with depression (AD-D) and 52 patients with $A D$ without depression (AD-nD). The amplitude of low frequency fluctuations (ALFF) was compared between two groups by performing independent-samples $t$-test. Results Compared with the AD-D group, increased ALFF values in the bilateral superior frontal gyrus, left middle frontal gyrus and left inferior frontal gyrus were observed in the AD-nD group. The brain activity in the AD-nD group in the bilateral superior frontal gyrus, left middle frontal gyrus and the left inferior frontal gyrus was higher than the AD-D group.

Conclusions Resting-state brain functional alterations may be closely bound up with the pathophysiologic features of patients with $A D$ with depressive symptoms.

\section{INTRODUCTION}

Alzheimer's disease (AD) is the most common type of dementia. The elderly population in China is estimated to reach 240 million in 2020. Currently, the prevalence of $\mathrm{AD}$ in this country is approximately $0.04 \%$ and the number of patients with $\mathrm{AD}$ is expected to reach 9 million. ${ }^{1}$ The prevalence of $\mathrm{AD}$ comorbid with depression is $42 \%$. $^{2}$ The comorbidity of $\mathrm{AD}$ and depression could impair cognitive function, increasing the burden of disease, leading to other comorbidities, therefore increasing the mortality of $\mathrm{AD} .^{3}$ Currently, the mechanisms of $\mathrm{AD}$ with depression remain unclear. A better understanding of the alterations in brain activity may help reveal the pathophysiological characteristics of patients with $\mathrm{AD}$ with depressive symptoms. Resting-state functional magnetic resonance imaging (rs-fMRI) based on blood oxygenation level dependent (BOLD) signal has become practical since the study by Biswal et al. ${ }^{4}$ This approach has been well performed in psychiatric disorders, such as depression, anxiety, $\mathrm{AD}$ and so on. ${ }^{5-7}$

Actually, based on the International Classification of Diseases (ICD) system, AD and depression belong to two independent spectrums of disease. Previous studies have suggested that $\mathrm{AD}$ is related with abnormal resting-state brain activity in the right medial frontal cortex, right inferior frontal gyrus, right postcentral gyrus and left anterior cingulate. ${ }^{7}$ Depression could affect resting-state brain activity in the left ventromedial prefrontal cortex, left inferior frontal gyrus, left precentral gyrus and the bilateral posterior cingulate and precuneus. ${ }^{8}$ These results demonstrated that $\mathrm{AD}$ and depression have different imaging markers in rs-fMRI. Previous resting-state functional MRI studies have found abnormal fractional amplitude of low frequency fluctuation (fALFF) changes in the fusiform gyrus, caudate nucleus and middle temporal gyrus ${ }^{9}$ as well as abnormal regional homogeneity in the right precentral gyrus, right superior frontal gyrus, right middle frontal gyrus and right inferior frontal cortex $^{10}$ in patients with $\mathrm{AD}$ with depression. These affected areas in $\mathrm{AD}$ with depression are different from both $\mathrm{AD}$ and depression, suggesting that there may be new pathological mechanisms and brain areas involved. For now, there is no consistent conclusion about the changes of fMRI brain activity in studies of the comorbidity of $\mathrm{AD}$ and depression.

We hypothesised that there were different brain activity regions between the patients with $\mathrm{AD}$ with and without depression in the resting state, and the abnormal brain function may be located primarily in the superior frontal gyrus, which is the cortex of emotional processing. Thus, we explored the difference of brain activity between the AD-D and AD-nD 


\section{Patients with Alzheimer's disease were recruited in the Zhejiang Provincial People's Hospital from October 2014 to October 2016. ( $\mathbf{N}=153)$}

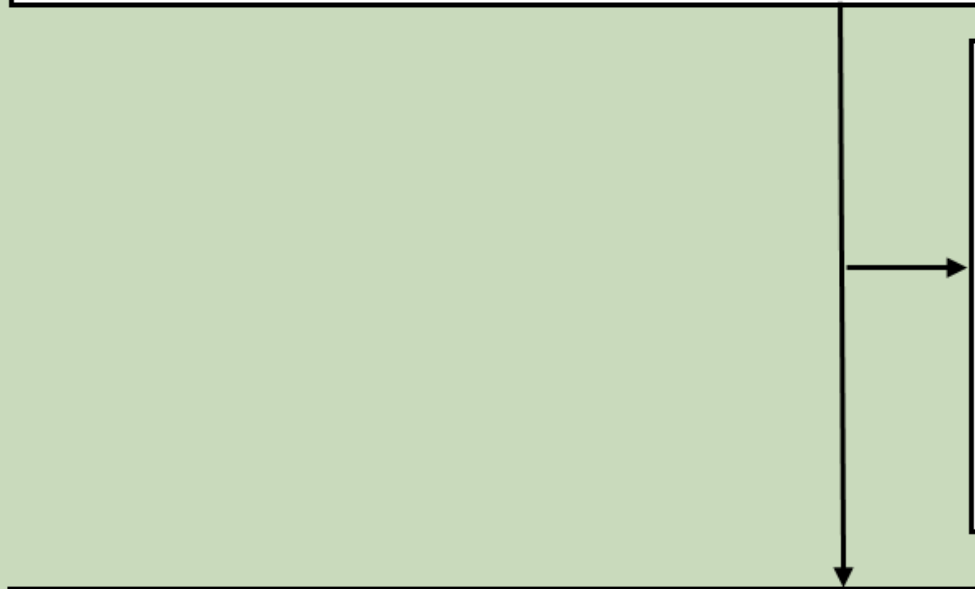

Excluded 79 subjects:

- Unmatched age $(\mathbf{N}=\mathbf{9})$

- With vascular dementia and intracranial tumors $(\mathrm{N}=13)$

- Poor image quality $(\mathrm{N}=18)$

- incomplete clinical data $(\mathrm{N}$

=12)

- head motion $(\mathrm{N}=27)$

\section{Patients with Alzheimer's disease in the study $(\mathbf{N}=74)$}

AD with depression $(\mathrm{N}=22)$

AD without depression $(\mathrm{N}=52)$

Figure 1 Flowchart of the study. AD, Alzheimer's disease.

groups, and the specific location of corresponding brain regions by the analysis of the amplitude of low frequency fluctuations (ALFF) in the resting-state functional MRI.

\section{METHODS}

Clinical information

The current study recruited patients with $\mathrm{AD}$ in the Zhejiang Provincial People's Hospital from October 2014 to October 2016. The inclusion criteria for AD were as

\begin{tabular}{|c|c|c|c|}
\hline & AD-D $(n=22)$ & AD-nD $(n=52)$ & $P$ value \\
\hline Mean (SD) age & $72.4(4.13)$ & $74.7(3.55)$ & 0.227 \\
\hline Gender (male/female) & $9 / 13$ & $29 / 23$ & 0.403 \\
\hline $\begin{array}{l}\text { Mean (SD) years of } \\
\text { education }\end{array}$ & $7.6(2.42)$ & $8.1(2.14)$ & 0.592 \\
\hline $\begin{array}{l}\text { Mean (SD) MMSE } \\
\text { score }\end{array}$ & 14.6 (3.08) & $15.8(2.20)$ & 0.213 \\
\hline $\begin{array}{l}\text { Mean (SD) HAMD-17 } \\
\text { score }\end{array}$ & $11.2(2.28)$ & $2.4(1.94)$ & $<0.001$ \\
\hline
\end{tabular}

Comparison of $A D$ with and without depression.

$A D$, Alzheimer's disease; $A D-D, A D$ with depression; AD-nD, AD without depression; HAMD-17, Hamilton Depression Rating Scale; MMSE, Mini-Mental State Examination. follows: (1) patients aged 65-80 years old; (2) educational attainment $\geq 6$ years; (3) exclusion of other major somatic diseases by chest X-ray and blood routine tests; (4) diagnosis by two psychiatrists with more than 10 years of clinical experience; (5) met the National Institute of Neurological and Communicative Disorders and StrokeAlzheimer's Disease and Related Disorders Association diagnostic criteria for $\mathrm{AD}$; (6) all the participants were right-handed; (7) scored under 26 on the Mini-Mental State Examination (MMSE). ${ }^{11}$ The participants were divided into the $\mathrm{AD}$ with depression group (AD-D) and $\mathrm{AD}$ without depression group (AD-nD) depending on whether they had core depressive symptoms.

The diagnostic criteria of $\mathrm{AD}$ with depression were: ${ }^{12}$ there was at least one core depressive symptom (depressive mood, loss of interest or pleasure) in the past 2 weeks and with a minimum of three of the following depressive symptoms: (1) depression (sadness, helplessness, crying and so on); (2) diminished interest and pleasure in social and daily life; (3) social disengagement or withdrawal; (4) loss of appetite; (5) insomnia; (6) decreased excitability of psychomotor activities (agitation or retardation); (7) easily irritated; (8) fatigue or lack of energy; (9) helplessness, self-blame; (10) repeated thoughts of death. These symptoms affect social function; delirium was excluded; somatic diseases or substance dependence were excluded, other mental disorders such as major depressive disorders 
and anxiety disorders were excluded. We evaluated the severity of depression by the 17-item Hamilton Depression Scale (HAMD) ${ }^{13}$ Exclusion criteria: (1) had vascular dementia and other types of dementia; (2) had other psychiatric diseases and a history of using psychotropic drugs before acquiring $\mathrm{AD}$; (3) unable to complete the imaging and psychosocial scale assessments; (4) scored less than 7 on the 17-item HAMD. All the subjects signed the informed consent form and the research protocol was approved by the Ethics Committee of Zhejiang Provincial People's Hospital.

\section{Measurement methods}

The Siemens Trio 3.0 T MR image with a dual phasedarray head coil was adopted. In the beginning, routine MRI scans including axial T1WI,T2WI,FLAIR followed by EPI-BOLD and 3DT1 imaging scans were used. Scanning parameters: EPI-BOLD sequence adopted, TR/TE $=2000 / 30 \mathrm{~ms}$, slice thickness $/ \mathrm{gap}=3.2 / 0.8 \mathrm{~mm}$, slice number $=31$, matrix size $=64 \times 64$, field of view $=220 \times$ $220 \mathrm{~mm}^{2}$, acquisition voxel size $=3 \times 3 \times 3 \mathrm{~mm}^{3}$, flip angle $=90^{\circ}$, time of scanning $484 \mathrm{~s}$. T1-weighted 3D-MPRAGE sequence: $\mathrm{TR} / \mathrm{TE}=8.5 / 3.2 \mathrm{~ms}$, slice thickness $=1 \mathrm{~mm}$, slice number $=176$, matrix size $=256 \times 256$, field of view $=$ $250 \times 250 \mathrm{~mm}^{2}$, voxel size $=1 \times 1 \times 1 \mathrm{~mm}^{3}$, flip angle $=15^{\circ}$, time of scanning $281 \mathrm{~s}$.

\section{Data preprocessing}

rs-fMRI data were processed using Data Processing Assistant for Resting-State fMRI toolkits ${ }^{14}$ including: (1) removing the first 10 time points to make the longitudinal magnetization reach steady state and to let the participant get used to the scanning environment; (2) slice-timing to correct the differences in image acquisition time between slices; (3) head motion correction; (4) spatial normalisation of the Montreal Neurological Institute (MNI) space via the deformation fields derived from tissue segmentation of structural images (resampling voxel size $=3 \times 3 \times 3 \mathrm{~mm}^{3}$ ); (5) spatial smoothing with an isotropic Gaussian kernel with a full width at half maximum of $6 \mathrm{~mm}$; (6) removing linear trend of the time course; (7) regressing out the head motion effect (using Friston 24 parameter) from the fMRI data; (8) band-pass filtering $(0.01-0.08 \mathrm{~Hz})$. A total of 27 participants were excluded from further analysis due to large head motion (more than $3.0 \mathrm{~mm}$ of maximal translation in any direction of $\mathrm{x}, \mathrm{y}$ or $\mathrm{z}$ or $3.0^{\circ}$ of maximal rotation throughout the course of scanning). Then, ALFF was further applied to the preprocessed fMRI data.

\section{ALFF calculation}

After data preprocessing, the time course for each voxel was transformed to the frequency domain with a fast Fourier transform and the power spectrum was then obtained. The square root was calculated at each frequency of the power spectrum and the averaged square root was obtained across $0.01-0.08 \mathrm{~Hz}$ at each voxel as the ALFF value, which was further divided by the global mean ALFF of each.

\section{Statistical analysis}

Independent sample $t$-test was used for comparing the difference of the ALFF values between the two groups. False discovery rate correction (FDC) was used for making corrections and it was thought to have statistical significance when $p<0.05$ and cluster volume $>3537 \mathrm{~mm}^{3}$. The results were read by the Viewer software of the Restplus software and the specific anatomical location of the brain regions with statistical significance in the MNI space was determined. The ALFF values were represented by the $t$ values, that $t>0$ was equivalent to escalated functional activity and $t<0$ was equivalent to a decreased functional activity.

\section{RESULTS}

\section{General demographics}

This research study recruited 153 patients in total. We excluded 9 cases who did not meet the age requirement, 13 cases who had vascular dementia and intracranial tumours, 18 cases who had poor image quality, 12 cases who had incomplete clinical data and 27 cases who were excluded from preprocessing due to large head motion. In the end, the data of 74 patients with $\mathrm{AD}$ were included for statistical analysis (figure 1). There was no difference of demographic data between the enrolled and excluded patients (online supplementary table 1 ). The patients were divided into the AD-D group $(\mathrm{n}=22)$ and $\mathrm{AD}-\mathrm{nD}$ group $(n=52)$ based on whether there were core depressive symptoms. All the patients were right-handed; the mean (SD) age of the two groups were: 72.4 (4.13) years old (AD-D), 74.7 (3.55) years old (AD-nD), respectively; gender ratio (male/female): 13/9 (AD-D), 29/23 (AD$\mathrm{nD}$ ); mean (SD) years of education: $7.6(2.42)$ years (AD$\mathrm{D}), 8.1$ (2.14) years (AD-nD); mean (SD) MMSE score: 14.6 (3.08) (AD-D), 15.8 (2.20) (AD-nD); mean (SD) Hamilton Depression Rating Scale (HAMD-17): 11.2 (2.28) (AD-D), 2.4 (1.94) (AD-nD). All the general demographic information had no statistical difference except HAMD-17 (table 1).

Table 2 Comparison of the ALFF values of the restingstate functional MRI between the AD with and without depression groups

\begin{tabular}{|c|c|c|c|c|c|c|}
\hline \multirow[b]{2}{*}{ Brain region } & \multirow[b]{2}{*}{ Side } & \multicolumn{3}{|c|}{ MNI coordinates } & \multirow[b]{2}{*}{ Voxels } & \multirow[b]{2}{*}{$T$ value } \\
\hline & & $x$ & $\mathbf{Y}$ & $\mathbf{Z}$ & & \\
\hline \multirow{2}{*}{$\begin{array}{l}\text { Superior frontal } \\
\text { gyrus }\end{array}$} & Left & -18 & 54 & 27 & 189 & 4.58 \\
\hline & Right & 16 & 46 & 33 & 247 & 4.72 \\
\hline $\begin{array}{l}\text { Middle frontal } \\
\text { gyrus }\end{array}$ & Left & -42 & 39 & 3 & 188 & 3.62 \\
\hline $\begin{array}{l}\text { Inferior frontal } \\
\text { gyrus }\end{array}$ & Left & -25 & 36 & -3 & 169 & 3.81 \\
\hline
\end{tabular}

AD, Alzheimer's disease; ALFF, amplitude of low frequency fluctuations. 


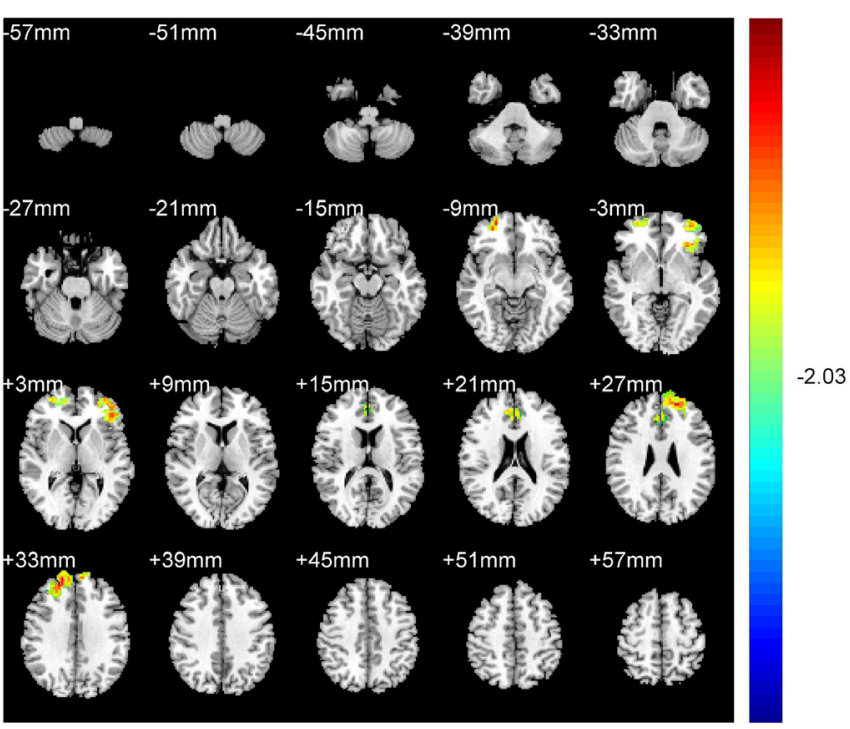

Figure 2 The $t$ value of each voxel is coded according to the proportion of the colour in the image. Compared with the $A D-D$ group, the brain regions with significantly higher ALFF values in the $A D-n D$ included the bilateral superior frontal gyrus, left middle frontal gyrus and left inferior frontal gyrus ( $p<0.05$, false discovery rate correction). AD, Alzheimer's disease; $A D-D, A D$ with depression; $A D-n D, A D$ without depression; ALFF, amplitude of low frequency fluctuations.

\section{Resting-state functional MRI in Alzheimer's disease with} depression

The ALFF values between the AD-D and AD-nD were compared by independent sample $t$ test. The brain regions with different $\mathrm{t}$ values were shown by sorting the voxel size $(p<0.05, \mathrm{FDC})$ : (1) bilateral superior frontal gyrus, (2) left middle frontal gyrus, (3) left inferior frontal gyrus. The ALFF values of the bilateral superior frontal gyrus, left middle frontal gyrus and the left inferior frontal gyrus in the $\mathrm{AD}-\mathrm{nD}$ group were significantly higher than those in the AD-D group (table 2, figure 2).

\section{DISCUSSION}

\section{Main findings}

In the current study, non-invasive and non-radioactive fMRI techniques were used to screen the biological markers of patients with $\mathrm{AD}$ and depression. Studies have found that there were differences in resting-state brain functional activities of the frontal lobe in patients with $\mathrm{AD}$ with depression. Compared with the AD-D group, the ALFF values of AD-nD group were significantly increased in the bilateral superior frontal gyrus, left middle frontal gyrus and left inferior frontal gyrus. It revealed that brain activities in the bilateral superior frontal gyrus, left middle frontal gyrus and left inferior frontal gyrus are low in patients with $\mathrm{AD}$ with depressive symptoms.

The dysfunction of the superior frontal gyrus is closely related to emotional dysfunction. It lacks the activation of the superior frontal gyrus in patients with depression during emotional stimulation. ${ }^{15}$ The level of decline of functional activities in the superior frontal gyrus is related to the severity of meditation. ${ }^{16}$ The decrease of functional activity of the superior frontal gyrus reflects the decrease of neurological activity and brain dysfunction in the corresponding regions. Guo $e t a l^{10}$ have indicated decline of functional activities of the right superior frontal gyrus, right middle frontal gyrus and right inferior frontal gyrus in patients with $\mathrm{AD}$ with depression. Therefore, changes of the superior frontal gyrus and middle frontal gyrus function could be one of the imaging markers of $\mathrm{AD}$ with depression. ${ }^{10}$ The inferior frontal cortex plays a key role in the executive function of the frontal-striato-circuit, especially in response to inhibition and cognition. ${ }^{1718} \mathrm{In}$ the state of depression, activities of the inferior frontal cortex decrease. ${ }^{19}$ The continuous low-grade brain activity in the inferior frontal gyrus may reflect the decrease of neuronal activity in the suppressed state. In the current study, the abnormal functional brain areas are located in the bilateral superior frontal gyrus, left middle gyrus and left inferior frontal gyrus, and the result is partially consistent with the study results obtained by the regional homogeneity analysis. ${ }^{10}$ Compared with the results of the regional homogeneity analysis, the number of brain areas with abnormal brain function was relatively small in the current study. The reason may be related to the variation in sensitivity of detecting abnormal brain activities in the two analytical methods because the two methods describe the different characteristics of neuronal electrical activity (excitability and synchronisation). The method of ALFF, which has high specificity, shows the intensity of spontaneous neuronal activities of the single voxel whereas the regional homogeneity method, which has high sensitivity, shows the synchronisation of spontaneous neuronal activities among multiple voxels, resulting in finding more abnormal brain activity areas than ALFF. ${ }^{20}$ The two methods have their own advantages and can be mutually complemental so that they are closer to the real results. These results suggest that low activity in the inferior frontal cortex may be a marker in depression. ${ }^{19}$

Carballedo et al found that the right hemisphere of the brain is essential for emotional processing, and it plays a leading role in affective processing ability compared with the left hemisphere. When the right side of the brain is damaged, the reduction of emotional processing ability becomes more apparent. ${ }^{21}$ However, the current study shows that the brain areas with decreased functional activity are mainly located in the left hemisphere. The reasons could be that all patients with $\mathrm{AD}$ are right-handed, or that the patients with $\mathrm{AD}$ have more severe brain atrophy in the local lobes on the basis of the whole brain atrophy. Atrophy in particular areas may trigger corresponding symptoms. Morphological voxel imaging and MRI have shown that the volume of grey matter decreased in the left middle frontal cortex $^{22}$ and medial temporal cortex ${ }^{23}$ in patients with $\mathrm{AD}$ with depressive symptoms. The mechanism of $\mathrm{AD}$ with depressive symptoms is complex and cannot be explained by a single specific neuron, nerve fibre or a specific brain area. The processing of emotional and cognitive information requires the integration of neurons and nerve fibres, 
that is, 'a small world network'. ${ }^{24}$ Therefore, according to the existing theory, the abnormal integration of neurons and nerve fibres, and abnormal brain network may also lead to the depressive symptoms of patients with AD. Diffusion tensor imaging studies of depression showed that there are abnormal functional connections in the postcentral gyrus, inferior temporal lobe and right insular lobe. ${ }^{25}$ In the current study, the ALFF values of the cingulate gyrus are slightly increased. Although this result is not as significant as other areas, the cingulate gyrus may also play an important role in the functional connectivity of the brain network. ${ }^{26}$ It is expected to have further exploration on the application of methods such as functional connectivity in brain network research in the future. In our study, we adopt ALFF analysis instead of fALFF to check the difference of brain regions between patients with $\mathrm{AD}$ with and without depression. Research showed that ALFF is strongly coupled with fALFF across voxels. ${ }^{27}$ They have been used to uncover differences in amplitude power both between subjects and between conditions, which showed moderate to high testretest reliability in grey matter regions. Both ALFF and fALFF analyses overlap, which could provide additional data to enable understanding of the disturbances of the related neural networks. ${ }^{28}$ The main difference between ALFF and fALFF is that ALFF is calculated as the sum of amplitudes within a specific low frequency range, while fALFF is calculated as a fraction of the sum of amplitudes across the entire frequency range detectable in a given signal. ALFF shows higher amplitude than fALFF as well as more sensitivity and reliability of these measures to grey matter than fALFF. ${ }^{27}$

\section{Limitations}

Certain limitations of the study should be considered when interpreting the results. First, there was a limited number of subjects due to difficulty in AD-D group enrollment. Second, physiological noise such as respiratory fluctuations may have influenced the stability of the resting-state fMRI signals during scanning. Third, we only compared ALFF in different brain regions between patients with $\mathrm{AD}$ with and without depression, but did not enroll healthy controls for our study. These limitations may lead to false-positive results. Thus, large-scale longitudinal studies are needed in the future for studying resting-state brain function in brain regions of patients with $\mathrm{AD}$ with depressive symptoms.

\section{Implications}

In conclusion, compared with the AD-D group, significant increased ALFF values in the bilateral superior frontal gyrus, left middle frontal gyrus and left inferior frontal gyrus were observed in the $\mathrm{AD}-\mathrm{nD}$ group, suggesting that these brain areas showed the increased functional activities of the AD-nD group. In addition, the exploration of the brain network function and conducting a task-based brain function study to patients with $\mathrm{AD}$ with depression could be conductive to revealing its related pathophysiological mechanism. These resting-state brain functional alterations are associated with pathophysiological characteristics of $\mathrm{AD}$ with depression, which may be used as a potential biological marker for patients with $\mathrm{AD}$ with depressive symptoms.

\section{Author affiliations}

${ }^{1}$ Department of Ultrasound, the First Affiliated Hospital, Zhejiang Chinese Medical University, Hangzhou, China

${ }^{2}$ Department of Radiology, Zhejiang Provincial People's Hospital, Hospital of Hangzhou Medical College, Hangzhou, China

${ }^{3}$ Department of Radiology, the First Affiliated Hospital, Zhejiang Chinese Medical University, Hangzhou, China

${ }^{4}$ Translational Medicine Research Center, Key Laboratory of Clinical Cancer Pharmacology and Toxicology Research of Zhejiang Province, Affiliated Hangzhou First People's Hospital, Zhejiang University School of Medicine, Hangzhou, China ${ }^{5}$ Department of Psychiatry, Yale University School of Medicine, New Haven, Connecticut, USA

${ }^{6}$ Shanghai Mental Health Center, Shanghai Jiao Tong University School of Medicine, Shanghai, China

Contributors YM wrote the article. QZ did the data analysis. MX and YL designed the experiment, $\mathrm{ZD}, \mathrm{MW}, \mathrm{XL}$ and $\mathrm{XG}$ reviewed and revised the article.

Funding This study was funded by Science and Technology Department of Zhejiang Province (2014C33126), Public Welfare Technology Research and Social Development Projects (2014-). This study is also funded by the National Natural Science Foundation of China (81201057), Shanghai Municipal Health Bureau Project (20124109), Chinese Medical Association, Psychiatry - Servier Youth Research Fund, Shanghai Mental Health Center international cooperation project (2013-) and Shanghai Municipal Center for Mental Health Clinical Research Program. This study is funded by Key Research Project of Zhejiang TCM Science and Technology Plan of China (2018ZZ010).

Competing interests None declared.

Patient consent for publication Not required.

Provenance and peer review Not commissioned; externally peer reviewed.

Data availability statement No data are available.

Open access This is an open access article distributed in accordance with the Creative Commons Attribution Non Commercial (CC BY-NC 4.0) license, which permits others to distribute, remix, adapt, build upon this work non-commercially, and license their derivative works on different terms, provided the original work is properly cited, appropriate credit is given, any changes made indicated, and the use is non-commercial. See: http://creativecommons.org/licenses/by-nc/4.0/.

\section{REFERENCES}

1 Li K, Wei S, Liu Z, et al. The prevalence of Alzheimer's disease in China: a systematic review and meta-analysis. Iran J Public Health 2018:47:1615.

2 Zhao Q-F, Tan L, Wang H-F, et al. The prevalence of neuropsychiatric symptoms in Alzheimer's disease: systematic review and metaanalysis. J Affect Disord 2016;190:264-71.

3 Novais F, Starkstein S. Phenomenology of Depression in Alzheimer's Disease. J Alzheimers Dis 2015;47:845-55.

4 Biswal B, Yetkin FZ, Haughton VM, et al. Functional connectivity in the motor cortex of resting human brain using echo-planar MRI. Magn Reson Med 1995;34:537-41.

5 Qiu H, Li X, Luo Q, et al. Alterations in patients with major depressive disorder before and after electroconvulsive therapy measured by fractional amplitude of low-frequency fluctuations (fALFF). J Affect Disord 2019;244:92-9.

6 Li K, Zhang M, Zhang $\mathrm{H}$, et al. The spontaneous activity and functional network of the occipital cortex is correlated with state anxiety in healthy adults. Neurosci Lett 2020;715:134596.

7 Liu X, Wang S, Zhang X, et al. Abnormal amplitude of low-frequency fluctuations of intrinsic brain activity in Alzheimer's disease. $J$ Alzheimers Dis 2014;40:387-97.

8 Wang L, Kong Q, Li K, et al. Frequency-dependent changes in amplitude of low-frequency oscillations in depression: a resting-state fMRI study. Neurosci Lett 2016;614:105-11.

9 Guo Z, Liu X, Li J, et al. Fractional amplitude of low-frequency fluctuations is disrupted in Alzheimer's disease with depression. Clin Neurophysiol 2017;128:1344-9.

10 Guo Z, Liu X, Jia X, et al. Regional coherence changes in Alzheimer's disease patients with depressive symptoms: a resting-state functional MRI study. J Alzheimers Dis 2015;48:603-11.

11 Folstein MF, Folstein SE, McHugh PR. "Mini-mental state". A practical method for grading the cognitive state of patients for the clinician. J Psychiatr Res 1975;12:189-98. 
12 Olin JT, Schneider LS, Katz IR, et al. Provisional diagnostic criteria for depression of Alzheimer disease. Am J Geriatr Psychiatry 2002;10:125-8.

13 Hamilton M. A rating scale for depression. J Neurol Neurosurg Psychiatry 1960;23:56-62.

14 Chao-Gan Y, Yu-Feng Z, Yan C. DPARSF: A MATLAB Toolbox for "Pipeline" Data Analysis of Resting-State fMRI. Front Syst Neurosci 2010;4:13.

15 Kupfer DJ, Frank E, Phillips ML. Major depressive disorder: new clinical, neurobiological, and treatment perspectives. Lancet 2012;379:1045-55.

16 Schiller CE, Minkel J, Smoski MJ, et al. Remitted major depression is characterized by reduced prefrontal cortex reactivity to reward loss. $J$ Affect Disord 2013;151:756-62.

17 Herwig U, Brühl AB, Kaffenberger T, et al. Neural correlates of 'pessimistic' attitude in depression. Psychol Med 2010;40:789-800.

18 Croxson PL, Johansen-Berg H, Behrens TEJ, et al. Quantitative investigation of connections of the prefrontal cortex in the human and macaque using probabilistic diffusion tractography. J Neurosci 2005;25:8854-66.

19 Hampshire A, Chamberlain SR, Monti MM, et al. The role of the right inferior frontal gyrus: inhibition and attentional control. Neuroimage 2010:50:1313-9.

20 Nimsky C, Kuhnt D, Ganslandt O, et al. Multimodal navigation integrated with imaging. Intraoperative imaging. Springer, 2011: 207-14.
21 Carballedo A, Scheuerecker J, Meisenzahl E, et al. Functional connectivity of emotional processing in depression. J Affect Disord 2011;134:272-9.

$22 \mathrm{Hu} \mathrm{X,} \mathrm{Meiberth} \mathrm{D,} \mathrm{Newport} \mathrm{B,} \mathrm{et} \mathrm{al.} \mathrm{Anatomical} \mathrm{correlates} \mathrm{of} \mathrm{the}$ neuropsychiatric symptoms in Alzheimer's disease. Curr Alzheimer Res 2015;12:266-77.

23 Tokuchi R, Deguchi K, Yamashita T, et al. [Impact of combined medial temporal atrophy and white matter lesions on the cognitive and emotional functions in Alzheimer's disease patients]. Nihon Ronen Igakkai Zasshi 2014;51:342-9.

24 Watts DJ, Strogatz SH. Collective dynamics of 'small-world' networks. Nature 1998;393:440-2.

25 Tadayonnejad R, Yang S, Kumar A, et al. Clinical, cognitive, and functional connectivity correlations of resting-state intrinsic brain activity alterations in unmedicated depression. J Affect Disord 2015;172:241-50.

26 Wang M, Liao Z, Mao D, et al. Application of Granger causality analysis of the directed functional connection in Alzheimer's disease and mild cognitive impairment. J Vis Exp 2017;126:e56015.

27 Zuo X-N, Di Martino A, Kelly C, et al. The oscillating brain: complex and reliable. Neuroimage 2010;49:1432-45.

28 Wang J-J, Chen X, Sah SK, et al. Amplitude of low-frequency fluctuation (ALFF) and fractional ALFF in migraine patients: a restingstate functional MRI study. Clin Radiol 2016;71:558-64.

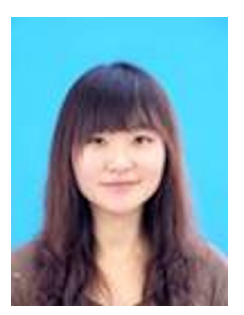

Yuzhu Mu obtained a bachelor's degree of medicine from Harbin Medical University in 2013. She was involved in the Residency Training Program of Zhejiang Province and practiced in the Zhejiang Provincial People's Hospital, Tongxiang Campus from 2013 to 2016. She has been working at the First Affiliated Hospital of Zhejiang Chinese Medical University since 2017. Her research interests include early diagnosis of Alzheimer's disease and neurosonography of fetal malformations. 